\title{
Empirical research on déjà vu experiences: a review
}

\author{
H.N. Sno, H.F.A. Schalken and F. de Jonghe \\ University Department of Psychiatry, Academic Medical Center, Amsterdam, The Netherlands \\ Correspondence to: H.N, Sno, Academic Medical Center, Psychiatric Outpatient Clinic, \\ Meibergdreef 9, 1105 AZ Amsterdam, The Netherlands
}

\begin{abstract}
A déjà vu experience is a dissociative phenomenon, which can be characterized as a subjectively inappropriate impression of familiarity of the present with an undefined past. This paper reviews empirical studies on déjà vu experiences and summarizes the most salient findings. Overall, the findings appear to be inconsistent and inconclusive. The authors conclude that the available empirical research is of limited significance due to various methodological and conceptual issues. In order to evaluate the clinical psychiatric relevance of déjà vu experiences, further research which also addresses its qualitative features is warranted.
\end{abstract}

Keywords: Clinical Psychiatry - Déjà vu experiences - Dissociative phenomena - Empirical Research

\section{INTRODUCTION}

In 1884 the American psychiatrist Henry Osborn was the first investigator who attempted to gather empirical evidence concerning what he called "illusive recognition" or "illusions of memory". For this purpose, he distributed a list of three questions at Princeton University: "Have you come suddenly upon an entirely new scene, and while certain of its novelty felt inwardly that you had seen it before-with a conviction that you were revisiting a dimly familiar locality? Mention if you can an instance or two in which this has occurred. Has any satisfactory explanation ever suggested itself to you?" Unfortunately Osborn failed to note the number of questionnaires he distributed, nor the response to them. All he noted of the findings was that approximately half the respondents answered affirmatively. He proceeded to discuss six characteristic descriptions given by his respondents and a number of déjà vu experiences that other authors had written about. On the grounds of his findings, he came to the conclusion that "the rationale of most illusive recognition, then, may be found in present analogies to the lost mental records of actual past life, in the former wanderings of our reveries and dreams, or in half conscious trains of thought" (p. 481).

To date these "illusions of memory" are generally known as déjà vu experiences. These experiences are characterized as any subjectively inappropriate impressions of familiarity of the present with an undefined past (Neppe, 1983). Most people are assumed to have had déjà vu experiences at one time or another. These ex- periences can occur in any sensory modality and may be viewed as misidentifications of time, place or person. Psychopathogetically déjà vu experiences can be related to disturbances or alterations in the normally integrative functions of identity, memory, perception or attention. Therefore, in terms of the DSM-III-R (APA, 1987), déjà vu experiences may be classified as dissociative phenomena (Sno and Linszen, 1990; Sno et al., 1992).

A subjective phenomenon like the déjà vu experience does not permit any experimental approach and the research on the subject is consequently almost exclusively committed to empirical studies. To our knowledge only three studies have provided some experimental evidence. By means of post-hypnotic amnesia, Banister and Zangwill (1941) succeeded to induce the feeling of familiarity, be it only in three of five subjects. Mullan and Penfield (1959) reviewed a series of 214 patients treated surgically for relief of their temporal lobe seizures. In six of these patients, it had been possible to reproduce a déjà vu experience by electrical stimulation of the temporal lobe. More recently Halgren et al. (1978) reported déjà vu experiences evoked by electrical stimulation of the median temporal lobe with stereotaxic placed depth electrodes in 5 of 36 psychomotor epileptics.

Ever since the study of Osborn (1884), only a small number of authors have attempted to gather empirical evidence concerning the déjà vu experience. The most comprehensive studies were performed by Bernard-Leroy (1898), Heymans (1904, 1906), Chapman and Mensh 
TABLE I. Empirical studies on déjà vu experiences

\begin{tabular}{|c|c|c|c|c|c|c|}
\hline Author & Year & Group studied & $\begin{array}{l}\text { Gender } \\
\mathrm{M} / \mathrm{F}\end{array}$ & Number & Interview & Questionnaire \\
\hline Osborn & 1884 & Princeton University & -1 & -1 & No & Self report \\
\hline Lalande & 1893 & -1 & $一^{1}$ & 100 & Yes & No \\
\hline Bernard-Leroy & 1898 & -1 & -1 & 67 & No & Self report \\
\hline Heymans & 1904 & Psychology students & $\begin{array}{l}M \\
F\end{array}$ & $\begin{array}{l}35 \\
10\end{array}$ & $\begin{array}{l}\text { No } \\
\text { No }\end{array}$ & $\begin{array}{l}\text { Self report } \\
\text { Self report }\end{array}$ \\
\hline Heymans & 1906 & Psychology students & $-^{1}$ & 88 & No & Self report \\
\hline $\begin{array}{l}\text { Chapman } \\
\text { and Mensh }\end{array}$ & 1952 & $\begin{array}{l}\text { Ambulatory and } \\
\text { clinical patients }\end{array}$ & $\begin{array}{l}M \\
F\end{array}$ & $\begin{array}{l}110 \\
110\end{array}$ & $\begin{array}{l}\text { Yes } \\
\text { Yes }\end{array}$ & $\begin{array}{l}\text { Observer } \\
\text { Observer }\end{array}$ \\
\hline $\begin{array}{l}\text { Richardson } \\
\text { and Winokur }\end{array}$ & 1967 & $\begin{array}{l}\text { Neurosurgical patients } \\
\text { Psychiatric patients }\end{array}$ & $\begin{array}{l}M \\
F \\
M \\
F\end{array}$ & $\begin{array}{r}93 \\
68 \\
117 \\
184\end{array}$ & $\begin{array}{l}\text { Yes } \\
\text { Yes } \\
\text { Yes } \\
\text { Yes }\end{array}$ & $\begin{array}{l}\text { Observer } \\
\text { Observer } \\
\text { Observer } \\
\text { Observer }\end{array}$ \\
\hline Harper & 1969 & Health Dept employees & $\begin{array}{l}M \\
F\end{array}$ & $\begin{array}{l}24 \\
67\end{array}$ & $\begin{array}{l}\text { Yes } \\
\text { Yes }\end{array}$ & $\begin{array}{l}\text { Observer } \\
\text { Observer }\end{array}$ \\
\hline Neppe & 1979 & Members Cultural Society & $\mathrm{F}$ & 84 & Yes & Observer \\
\hline Neppe & 1983 & $\begin{array}{l}\text { "Normals" } \\
\text { "Neuropsychiatric" patients }\end{array}$ & $-^{1}$ & $\begin{array}{l}28 \\
48\end{array}$ & $\begin{array}{l}\text { Yes } \\
\text { Yes }\end{array}$ & $\begin{array}{l}\text { Observer } \\
\text { Observer }\end{array}$ \\
\hline
\end{tabular}

1 Information not provided.

(1952), Richardson and Winokur (1967, 1968), Harper (1969) and Neppe $(1979,1983)$ (Table I). In this paper we review the studies by these authors in more detail. In the conclusion, the most salient findings as well as the consequences as to future research are discussed.

The study of Bernard-Leroy (1898)

In 1898 Bernard-Leroy, a French psychiatrist, published his dissertation describing the findings of his research on the déjà vu experience. His study was conducted with a self-administered questionnaire that had been previously published in an English-language and a French-language journal. This questionnaire included 36 questions on the frequency of the déjà vu experiences, and on predisposing and precipitating conditions. He received only 67 completed questionnaires, and 49 of these were included unedited at the end of his dissertation. Due to this low response rate, Bernard-Leroy was not able to draw any conclusion as to the frequency of occurrence.

In addition to this questionnaire study, a large number of subjects were either interviewed by Bernard-Leroy himself or by one of his colleagues. About half of the approximately 500 non-disturbed individuals who were interviewed had had déjà vu experiences. Of some 60 patients at the Salpêtrière, only six appeared to have experienced the phenomenon. Due to incoherence, the reliability of these patients was limited. Bernard-Leroy nonetheless concluded that the estimate of $30 \%$ by Lalande (1893), based on interviews with 100 individuals, probably came closest to the truth. Based on his own findings, Bernard-Leroy felt he could conclude that a déjà vu experience was generally accompanied to varying degrees by a clearly painful or pleasant effect or by depersonalization.
The frequency rate was not assumed to be influenced by gender, race or social-economic status. Bernard-Leroy did however feel there were indications that age played a role. On the basis of his interviews, he could not confirm the facilitating influence of fatigue, intoxication, infectious disease or emotional states. In his opinion, the influence of epilepsy was overestimated; none of the 98 epileptic patients he interviewed at the Salpêtrière had experienced the phenomenon. Moreover, déjà vu experiences did not prove to occur any more frequently among so-called "neuropathic" patients than among so-called "normal" individuals.

Heymans' studies $(1904,1906)$

In 1904, the Dutch psychologist Heymans reported the findings of a prospective study on déjà vu experiences and depersonalization. The study was performed by using a 15-item questionnaire, with which Heymans attempted to elucidate characteristic personality traits as well as specific facilitating conditions. The sample consisted of 35 male and 10 female psychology students in the 20-25 age range. The response rate was $93 \%$ and $41 \%$ of the respondents claimed to have had at least one déjà vu experience at one time or another. Eight of his respondents reported a total of 13 déjà vu experiences during the research period.

Without any verifiable statistical analysis, Heymans felt that on the grounds of his data, he could specify four personality traits as predisposing factors: emotional sensitivity, mood fluctuations, periods of apathy and an irregular working rhythm. In his view, this conclusion was confirmed by the findings of a replication study published in 1906. The respondents $(n=88)$ of the 1906 study were sampled by way of a request in the 1904 article, and from psychology students of a different year's course. This time 
the life-time prevalence of déjà vu experiences amounted to $63 \%$. Thirty-one of the 88 respondents prospectively reported a total of 94 déjà vu experiences.

To our knowledge, Heymans' study is unique in that it is the only study with a prospective design. The disadvantage of the possible overinclusiveness of this design was refuted by Heymans in advance. He argued that déjà vu experiences were based on disturbances of attention. Consequently, focusing extra attention on the phenomenon would be more apt to lead to under-reporting.

The findings of his study did not yield the desired insight into links between déjà vu experiences and specific facilitating conditions. Heymans did however tentatively conclude, that the findings of the 1904 study and of the combined groups, supported the notion of a link with conditions accompanied by a temporary reduction of psychological energy. Heymans observed that déjà vu experiences occurred more frequently than depersonalization, whereas predisposing factors were most pronounced in respondents with depersonalization. In his view these findings confirmed his theory that déjà vu experiences and depersonalization were manifestations of one and the same process. Both of these phenomena were based on reduced psychological energy or a reduced ability to concentrate attention, so that those associations that would add a quality of familiarity would be absent or abnormally weak. He worked from the assumption that the intensity and the number of associations may serve as a measurement for stipulating the dating in the past: the vaguer and more insignificant the associations, the more time has passed between the present and the remembered event. In depersonalization, the quality of familiarity is absent. Déjà vu experiences are thought to emerge when the associations are not completely absent, but are weak and few in number. In the individual's consciousness, there is then the illusion that the present event is actually a memory from the very distant past.

\section{The study of Chapman and Mensh (1952)}

Using an original questionnaire they had developed themselves, Chapman and Mensh (1952) interviewed 220 white ambulatory clinical patients in the Washington University Clinics. Their study was designed as a preliminary attempt to obtain more information on déjà vu experiences and daydreaming. The questionnaire included questions on socio-demographic features and frequency of traveling, daydreaming and déjà vu experiences. The sample largely consisted of patients with 4-12 years of education $(80 \%)$ and skilled or semi-skilled workers or housewives (70\%); $92 \%$ of them traveled more than 50 miles a year. The incidence of déjà vu experiences was $35 \%$ among the females and $31 \%$ among the males. As to frequency per person, there were similarly no significant differences. There were, however, indications that persons with a higher occupational level had déjà vu experiences more often. In addition, people who never traveled had them less often than frequent travelers: $11 \%$ as compared to $24-44 \%$. As regards age and education, a negative and a positive correlation were observed respectively.

\section{The studies of Richardson and Winokur (1967, 1968)}

Richardson and Winokur $(1967,1968)$ used the same questionnaire as Chapman and Mensh (1952). Over a 3-month period they interviewed 161 consecutive admissions to a large neurosurgery service of Barnes Hospital in St Louis and 301 consecutive admissions to a large university psychiatric hospital of Renard Hospital, St Louis. The incidence of déjà vu experiences was $40 \%$ and $44 \%$ respectively. Like Chapman and Mensh, they also found indications of a correlation with educational level and occupation. The correlation with traveling could only be confirmed in the group of neurosurgery patients. Differences between men and women did not appear to be significant. A link to race was noted in that white respondents had déjà vu experiences more often than black ones.

Neither psychiatric nor cerebral pathology was evident in 73 of the total of 462 patients and of these 73 patients, 21 or $30 \%$ reported having had a déjà vu experience. Of the 289 patients solely diagnosed as having a psychiatric disorder, 140 or $48 \%$ reported having had a déjà vu experience, as did 20 or $42 \%$ of the 47 patients solely diagnosed as having a cerebral disorder. These differences were felt to be significant $(p<0.05)$.

In the group of psychiatric patients, the déjà vu experience appeared to occur significantly more frequently in the categories of personality disorders and mixed diagnoses $(p<0.05)$. The disorders in the first category included inadequate, emotionally unstable, passiveaggressive, sociopathic, schizoid and sexually deviant personalities. The mixed diagnoses included mental retardation, situational reactions and adjustment disorders of adolescents. The incidence of $42 \%$ among patients with a depression was lower than the average of $44 \%$ and the incidence of $54 \%$ among patients with schizophrenia was higher. Upon closer examination of the category of psychoneuroses, the déjà vu experience appeared to occur relatively frequently, although not significantly so, among patients with a hysterical personality (57\%) and anxiety neuroses $(56 \%)$. On the basis of these findings in the psychiatric group, the authors suggested that the phenomenon of déjà vu may be of help in clarifying the vague psychiatric diagnostic categories called personality disorders and situational reactions.

In the neurosurgery group of patients, déjà vu experiences appeared to be equally divided among the various diagnostic categories. On the grounds of this finding, Richardson and Winokur tentatively concluded that 
déjà vu experiences do not occur any more frequently in patients with temporal epilepsy than in patients with or without other types of pathology.

Harpers' study (1969)

In 1969, Harper reported the findings of a study in which 91 employees at a municipal public health department were interviewed, varying in age from 18 to 65 . The prevalence of the déjà vu experience was $62.6 \%$. No correlation with age and gender could be observed. The majority of the research population could not stipulate any specific circumstances. Harper noted as a possible trend that déjà vu experiences occurred less frequently among persons with "marked neurotic features". Persons with compulsive features were, however, observed to have déjà vu experiences more frequently, although not significantly so. The respondents who reported having had déjà vu experiences were less "emotionally sensitive". Déjà vu experiences occurred significantly more frequently among respondents who reported depersonalization. There was no significant correlation, but a trend was observed correlating with higher intelligence and social class, Harper concluded: "Those reporting déjà vu are not likely to be more psychiatrically unhealthy nor to have more seriously disturbed personalities than those not reporting déjà vu, indeed the reverse may be true, but the presence of obsessional traits and occupational and intellectual status likely to be associated with verbal skills may facilitate the identification and reporting of this elusive phenomenon." As regards temporal epilepsy, Harper noted that it was impossible to detect any difference between an ordinary déjà vu experience and an epilepsy-related déjà vu experience.

\section{The studies of Neppe $(1979,1983)$}

In 1979, Neppe published the findings of a study performed with a questionnaire he had developed himself. The research population consisted of 84 universitytrained, white female members of a cultural society ranging in age from 40 to 70 . The life-time prevalence was $96 \%$. Neppe concluded that the findings indicated a correlation of déjà vu experiences with educational level, social status, intelligence and cultural interest.

In his dissertation in 1983, Neppe reported the findings of a pilot study on qualitative aspects and the clinical significance of déjà vu experiences. The study was performed with a structured interview based on a number of questionnaires he had developed himself. The research population consisted of 28 "normal" respondents and 48 "neuropsychiatric" patients at an outpatient clinic. As a result of the selection procedure and demographic differences, it was not possible to compare the "normal" respondents and the "neuropsychiatric" patients. In the "normal" group, Neppe compared 17 respondents who had never had any paranor- mal experiences (subjective paranormal non-experience-SPNE) with six respondents who had at some point in their lives had experiences of this kind (subjective paranormal experience-SPE). The remaining "intermediate" group of five respondents was left out of consideration. In the neuropsychiatric group, 20 patients with schizophrenia according to DSM-III (APA, 1980) and Research Diagnostic Criteria (Feighner et al., 1972), 14 with temporal epilepsy (TLE) and 14 with non-temporal epilepsy (NTE) were compared.

In comparing the life-time prevalence in the "normal" sub-groups, déjà vu experiences appeared to occur more frequently in the SPE sub-group (100\%) than in the SPNE sub-group $(47 \%)(p<0.01)$. In contrast to the schizophrenic patients and the SPE sub-group, in the SPNE subgroup the déjà vu experiences had had very little emotional impact. In the "normal" group, remembering dreams proved to be a necessary but in itself inadequate pre-condition for the occurrence of déjà vu experiences. No correlation with age was observed.

In the "neuropsychiatric" group, the TLE sub-group was compared with the schizophrenic patients. The lifetime prevalence of déjà vu experiences proved to be significantly higher in the TLE sub-group (86\%) than in the schizophrenic sub-group $(65 \%)(p<0.01)$.

The TLE sub-group could be further divided into a group of patients who frequently had déjà vu experiences and a group who seldom did. A comparison with the NTE sub-group showed that a number of symptoms also occurred more frequently in the TLE sub-group, such as postictal features $(p<0.001)$, jamais vu $(p<0.01)$, a heightened awareness of one's own body $(p<0.01)$ and influence on thinking $(p<0.01)$. According to Neppe, this might indicate that these symptoms are specific to temporal epilepsy. The TLE sub-group exhibited the highest frequency of déjà vu experiences per individual $(p<0.05)$. For $80 \%$ of the TLE respondents and the patients with temporal dysfunction in the NTE sub-group, the form of the déjà vu experience had always been the same. On the basis of this finding, Neppe suggested that déjà vu experiences that always assume the same form might be an indication of temporal epilepsy. In the TLE sub-group, depersonalization occurred more frequently than in the other groups.

In the TLE sub-group, déjà vu experiences occurred all the more frequently if and when medicinal treatment was not optimal. These symptoms could thus be utilized as a signal in the course of the treatment. In addition, déjà vu experiences could contribute toward the prevention of insults if patients were to recognize these symptoms as auras.

The déjà vu experiences of the NTE patients bore marked similarities to those of the "normal" group without paranormal experiences (SPNE). In schizophrenic 
patients, the déjà vu experience was often related to places and to reading. In this group, the questionnaries revealed a number of disorders that could serve as indications of schizophrenia. This led Neppe to conclude that his questionnaires could have a diagnostic value. A slight correlation was noted between the occurrence of déjà vu experiences and inadequate medicinal treatment of schizophrenic symptoms. According to Neppe, a déjà vu experience generally lasts several seconds. In schizophrenic patients, however, the duration can fluctuate. The majority of the total population was not able to give any explanation for the déjà vu experience, nor were they able to specify any decisive precipitating factors. Fatigue, anxiety and stress played a role to varying extents.

On the grounds of his findings, Neppe concluded that four sub-types of déjà vu experiences could be qualitatively distinguished: the temporal epileptic, the schizophrenic, the subjective paranormal and the associative déjà vu experience.

The temporal epileptic déjà vu experience is characterized by the presence of postictal features (e.g. headache, fatigue, somnolence) or actual blackouts or fits immediately following the experience. This experience may also be extremely frequent, of prolonged duration, very clear, and emotionally and cognitively intense. It appears to be having "exactly the same" form every time, and to be associated with heightened awareness of the environment and one's own body.

The "schizophrenic" déjà vu experience is characterized by the presence of psychotic thinking and incongruous affect. Since these features are not specific to schizophrenia, Neppe suggested using the term "psychotic déjà vu experience".

The "subjective paranormal" déjà vu experience is distinguished by subjective paranormal experiences: precognition and retrocognition, marked familiarity, "experiential growth", extreme clarity, marked cognitive change, polymodal perception, heightened awareness of the environment, altered awareness of self, and usually euphoric but congruous affective experience. There is usually a marked sense of conviction and the experience is described as "sensed".

The associative déjà vu experience probably reflects the average kind of déjà vu experience in the general population. This subtype is sometimes evoked by certain actions or environment stresses. The experience is generally not very clear, the familiarity is slight, and it is poorly remembered. It generally lasts a few seconds and is associated with little or no emotional and cognitive change.

\section{CONCLUSIONS}

To date the findings of empirical research on déjà vu experiences have been inconsistent and inconclusive. The reported rates vary from 30 to $96 \%$. There would seem to be a predilection for the younger age groups. However, neither Lalande (1893), Neppe (1983) nor Harper (1969) noted any correlation with age. The majority of authors reported some extent of correlation with depersonalization. However, in a study on depersonalization Braeuer $e t$ al. (1970) found that déjà vu experiences correlated with just a few of the variables depersonalization and derealization were correlated with. With the exception of the gender factor, no unequivocal correlations could be noted with such demographic variables as educational level, socio-economic status or race. In contrast to the findings of Bernard-Leroy (1898), Lalande (1893) and Harper (1969), Richardson and Winokur (1968) concluded that déjà vu experiences occurred significantly more frequently among psychiatric patients. Neppe felt that déjà vu experiences had a diagnostic value with respect to epilepsy, although Bernard-Leroy (1898) and Harper (1969) were convinced this was not the case.

The methodological and conceptual issues in the above empirical studies make interpretation and comparison of their findings tentative. None of the questionnaires used in these studies have been submitted to basic statistical analyses to establish the reliability or validity of these instruments. The reporting style used by Osborn (1884), Lalande (1893) and Bernard-Leroy (1898) was impressionistic and incomplete. Heymans' study might indeed have been prospective, but due to the selection procedure he used, his research population was not representative. The study by Richardson and Winokur (1967, 1968) examined the correlation between déjà vu experiences and psychiatric diagnoses on the basis of archaic diagnostic categories.

The majority of the studies relied primarily on a dichotomous format, i.e. déjà vu experiences were either present or absent. With the exception of the studies performed by Neppe (1979, 1983), none of the studies included any questions on the qualitative aspects of déjà vu experiences. However, the value of Neppe's study is limited because his data are based on instruments administered by only one interviewer and on a small sample. In addition, Neppe's selection procedure and demographic differences of his respondents made it unfeasible to compare the separate sub-groups.

In short, empirical research on déjà vu experiences is scanty and the findings are of limited significance. To date the evaluation of the clinical relevance of déjà vu experiences has been inadequate and further research that also addresses its qualitative features is warranted. The present authors are currently preparing a study of this kind using a self-administered questionnaire they have devised.

\section{REFERENCES}

American Psychiatric Association (1980) Diagnostic and Stat- 
istical Manual of Mental Disorders, 3rd Edn. APA, Washington, DC.

American Psychiatric Association (1987) Diagnostic and Statistical Manual of Mental Disorders, 3rd Edn. Revised. APA, Washington, DC.

Banister H and Zangwill OL (1941) Experimentally induced visual paramnesias. British Journal of Psychology, 32, 30-51.

Bernard-Leroy E (1898) L'illusion de Fause Reconnaissance. F. Alcan, Paris.

Brauer R, Harrow M and Tucker GJ (1970) Depersonalization phenomena in psychiatric patients. British Journal of Psychiatry, 117, 509-515.

Chapman AH and Mensh IN (1951/1952) Déjà-vu experience and conscious fantasy in adults. Psychiatric Quarterly, Suppl. 25-26, 163-175.

Feighner JP, Robins E, Guze SB et al. (1972) Diagnostic criteria for use in psychiatric research. Archives of General Psychiatry, 26, 57-63.

Halgren E, Walter RD, Cherlow DG and Crandall PH (1978) Mental phenomena evoked by electrical stimulation of the human hippocampal formation and amygdala. Brain, 101, 83-117.

Harper MA (1969) Déjà vu and depersonalization in normal subjects. Australian and New Zealand Journal of Psychiatry, 3, 67-74.

Heymans G (1904) Eine Enquête über Depersonalisation und "Fausse Reconnaissance". Zeitschrift für Psychologie, 36, 321-343.

Heymans G (1906) Weitere Daten über Depersonalisation und
"Fausse Reconnaissance". Zeitschrift für Psychologie, 43, $1-17$

Lalande A (1893) Des paramnésies. Revue Philosophique de la France et de l'etranger, 36, 485-497.

Mullan S and Penfield W (1959) Illusions of comparative interpretation and emotion. Archives of Neurology and Psychiatry, 81, 269-284.

Neppe VM (1979) An investigation of the relationship between subjective paranormal experience and temporal lobe symptomatology. Dissertation. University of the Witwatersrand, Johannesburg.

Neppe VM(1983) The Psychology of Déjà Vu: Have I Been Here Before? Witwatersrand University Press, Johannesburg.

Osborn HF (1884) Illusions of memory. The North American Review, 138, 476-486.

Richardson TF and Winokur G (1967) Déjà vu in psychiatric and neurosurgical patients. Archives of General Psychiatry, 17, 622-625.

Richardson TF and Winokur G (1968) Déjà vu—as related to diagnostic categories in psychiatric and neurosurgical patients. Journal of Nervous and Mental Diseases, 146, 161-164.

Sno HN and Linszen DH (1990) The déjà vu experience: remembrance of things past? American Journal of Psychiatry, 147, 1587-1595.

Sno HN, Linszen DH and De Jonghe F (1992) Déjà vu experiences and reduplicative paramnesia. British Journal of Psychiatry, 160, in press. 


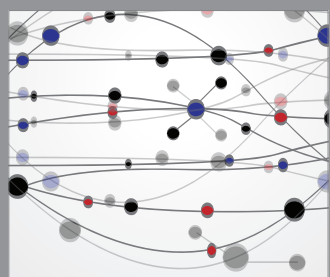

The Scientific World Journal
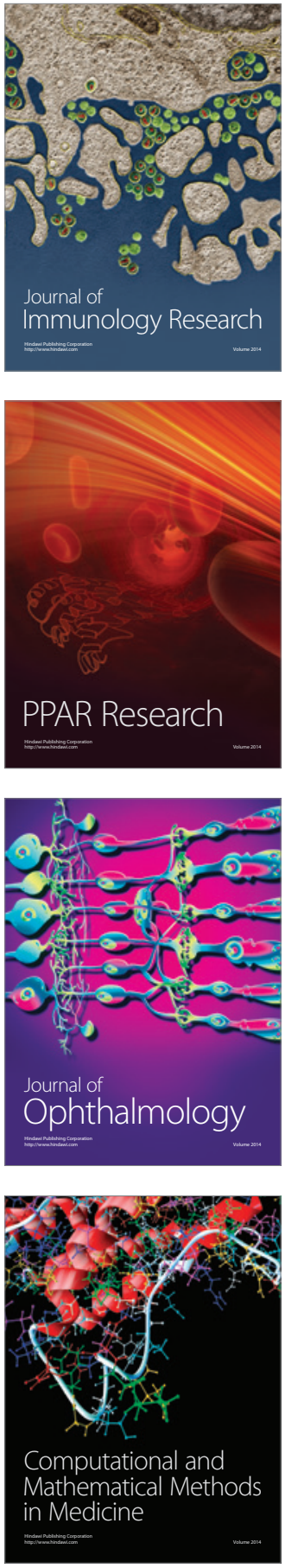

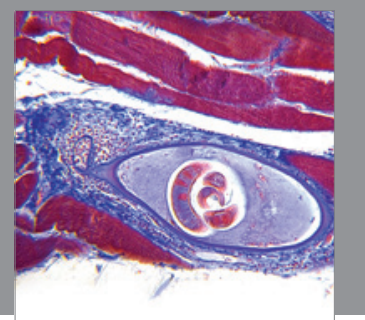

Gastroenterology

Research and Practice
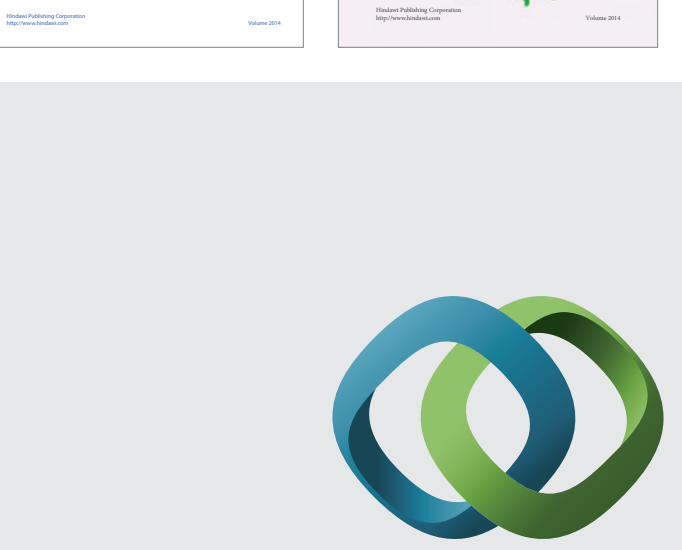

\section{Hindawi}

Submit your manuscripts at

http://www.hindawi.com
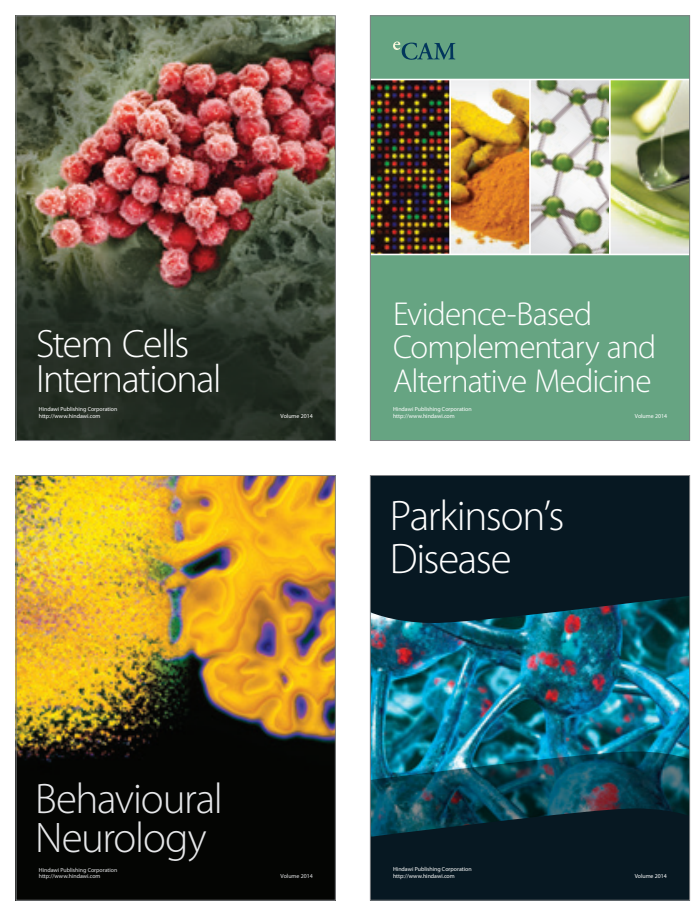

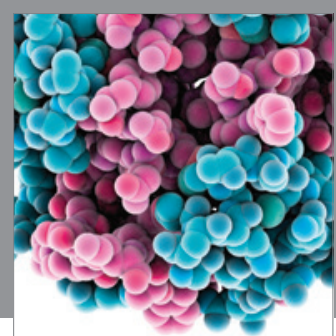

Journal of
Diabetes Research

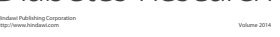

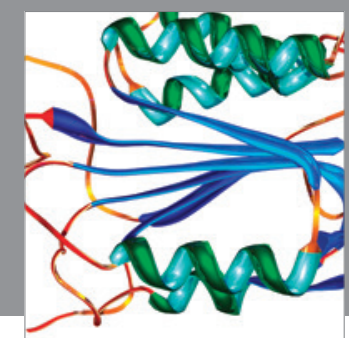

Disease Markers
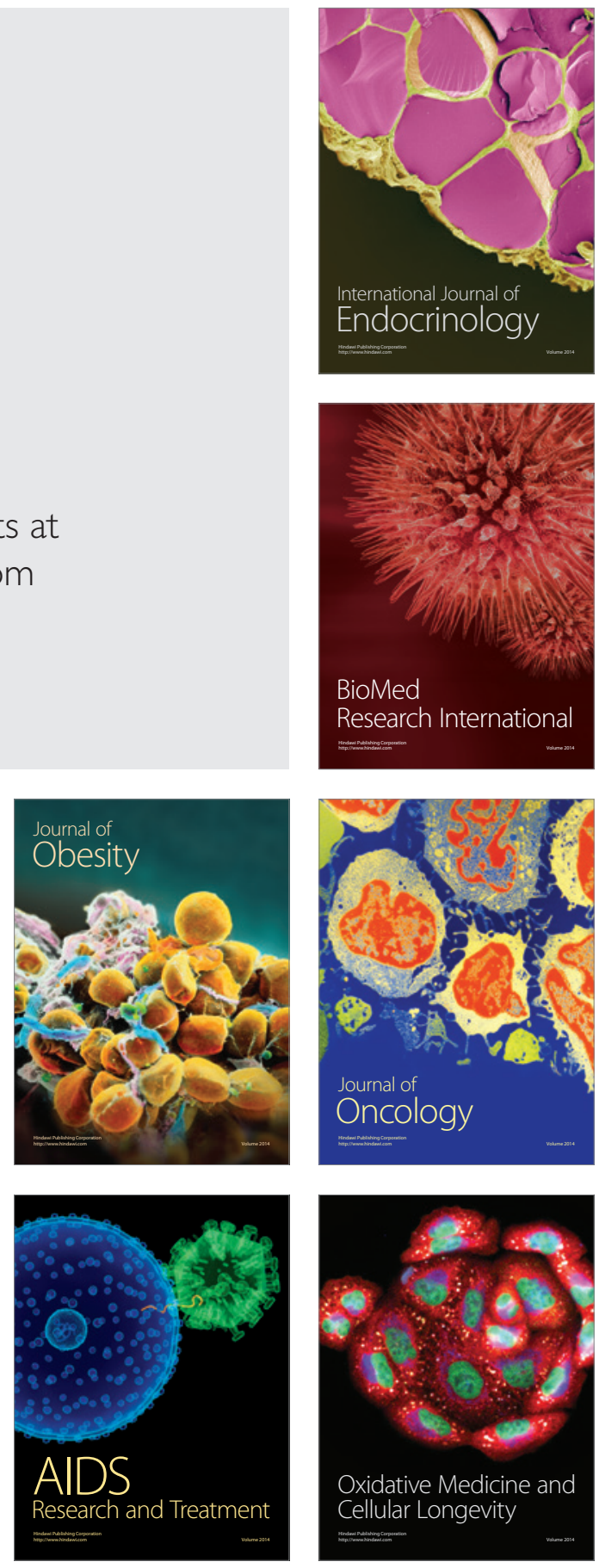